\title{
Perpendicular Magnetic Anisotropy and Intralayer Interactions in a Single Layer of CoPt Nanoparticles
}

\author{
J. Rubín ${ }^{1, a, *}$, A. I. Figueroa ${ }^{2}$, J. Stankiewicz ${ }^{2}$, F. Bartolomé ${ }^{2}$, L.M. García ${ }^{2}$, \\ J. C. Cezar ${ }^{3}$, F. Petroff ${ }^{4}$, C. Deranlot ${ }^{4}$ and J. Bartolomé ${ }^{2}$ \\ ${ }^{1}$ Instituto de Ciencia de Materiales de Aragón, CSIC-Universidad de Zaragoza, Dept. Ciencia y \\ Tecnología de Materiales y Fluidos, E-50018, Zaragoza, Spain \\ ${ }^{2}$ Instituto de Ciencia de Materiales de Aragón, CSIC-Universidad de Zaragoza, Dept. Física de la \\ Materia Condensada, E-50009, Zaragoza, Spain \\ ${ }^{3}$ European Synchrotron Radiation Facility CS40220, F-38043 Grenoble Cedex 9, France \\ ${ }^{4}$ Unité Mixte de Physique, CNRS, Thales, Université Paris-Sud, Université Paris-Saclay, 91767, \\ Palaiseau, France \\ a jrubin@unizar.es
}

Keywords: single layers, nanoparticles, Co, Pt, magnetic properties.

\begin{abstract}
The multilayer films $\left[\mathrm{Al}_{2} \mathrm{O}_{3} / t_{\mathrm{Co}} \mathrm{Co} / t_{\mathrm{Pt}} \mathrm{Pt}\right]_{\mathrm{N}}$, produced by sequential deposition of Co and $\mathrm{Pt}$ on alumina consist in layers of CoPt alloyed nanoparticles. They show perpendicular magnetic anisotropy (PMA) below a freezing temperature $T_{\mathrm{f}}$, an asperomagnetic-like phase below that temperature, and hard ferromagnetic ordering below a transition temperature $T_{1}<T_{\mathrm{f}}$. A single layer granular film $(N=1)$ with deposition thicknesses $t_{\mathrm{Co}}=0.7 \mathrm{~nm}, t_{\mathrm{Pt}}=1.5 \mathrm{~nm}$ and particle diameter of 3 $\mathrm{nm}$ is presently studied. SQUID magnetometry shows that a single layer presents the three phases as well. Para-, aspero- and ferromagnetic phases are observed upon lowering the temperature, with transition temperatures $T_{\mathrm{f}} \approx 375 \mathrm{~K}$ and $T_{1} \approx 200 \mathrm{~K}$, respectively. In addition, the PMA persists, proving that there is no interlayer coupling in the multilayer system. SQUID results also reveal a core-shell structure in the CoPt nanoparticles.
\end{abstract}

\section{Introduction}

Understanding the magnetic anisotropy of assembled nanoparticles (NPs) systems is central to develop their possible technological applications. Towards this end, an intense research of various cobalt granular systems has been carried out for more than a decade by exploring enhanced magnetic anisotropy [1-6]. Those granular films consist of self-organized lattices of nanometric Co magnetic clusters (1-4 nm in diameter) prepared by sequential deposition of $\mathrm{Al}_{2} \mathrm{O}_{3}$ and Co layers on a Si substrate. Magnetic anisotropy [2,3] and dipolar interactions [1] have been determined, and the surface anisotropy has been controlled by capping the cobalt NPs with noble metals $\mathrm{M}(\mathrm{M}=\mathrm{Cu}$, $\mathrm{Ag}$, and $\mathrm{Au}$ ) [4], which do not alloy with the Co NP, but simply surround them.

An alternative approach to control the anisotropy of these Co granular systems is to produce alloyed particles in order to modify the intrinsic source of anisotropy. In particular, granular films of Co capped with W [6], Pt [7-8] and Pd [9,10] have been studied. The multilayer films of CoPt alloyed nanoparticles, produced by sequential deposition of $\mathrm{Co}$ and $\mathrm{Pt}$ on alumina show $\mathrm{CoPt}$ clusters with perpendicular magnetic anisotropy (PMA) below a freezing temperature $T_{\mathrm{f}}$, soft ferromagnetic ordering below that temperature, and hard ferromagnetic ordering below a crossover temperature $T_{1}<T_{\mathrm{f}}$. The question assessed in the present work is whether an interlayer interaction plays any role in the establishment of the ordered phase below $T_{\mathrm{f}}$ and in the crossover below $T_{1}$, as was considered in Ref. [8]. To this end a single layer granular film was grown on Si with an alumina buffer to exclude interlayer coupling. The nominal Co and Pt thicknesses were $t_{\mathrm{Co}}=0.7 \mathrm{~nm}$ and $t_{\mathrm{Pt}}=1.5 \mathrm{~nm}$, which produced $3 \mathrm{~nm}$ diameter NPs. A protective alumina layer was deposited on top of the CoPt layer. 


\section{Experimental}

Magnetization measurements were performed with a SQUID magnetometer equipped with the high-resolution (RSO) option. Hysteresis loops at several temperatures and zero-field cooling (ZFC) and field cooling (FC) dc susceptibility as a function of temperature with a field of $0.2 \mathrm{kOe}$ applied parallel and perpendicular to the substrate plane were measured. Anomalous Hall effect (AHE) measurements were carried out in a home made electrical transport measurement setup and in a Quantum Design Physical Property Measurement System (PPMS). A six-probe method was employed with the contacts bonded by wire welding and micro-contacting at the edges of the rectangular samples. The magnetic field was varied within $H= \pm 20 \mathrm{kOe}$, and applied in the direction perpendicular the substrate. $V_{\mathrm{H}}$ versus $H$ loops were collected at several temperatures from 2 to $300 \mathrm{~K}$. X-ray magnetic circular dichroism (XMCD) magnetization loops were obtained from the $\mathrm{Co} L_{3}$ edge fluorescence yield (FY) spectra at the ESRF ID08 beamline.

\section{Results and discussion}

The FC and ZFC curves of the susceptibility $\chi$ for applied magnetic field parallel $(\|)$ and perpendicular $(\perp)$ to the substrate's plane are displayed in Fig. 1, together with the same type of measurements for the multilayer $\left[\mathrm{Co} / \mathrm{Pt} / \mathrm{Al}_{2} \mathrm{O}_{3}\right]_{N}$ with $N=25$ [8]. To enable a comparison between the single layer and the multilayer, $\chi$ is shown in emu/ $\mathrm{cm}^{3}$ of deposited Co. As temperature increases, the single layer shows the same sequence of magnetic phases as in the multilayer:

i) A low temperature hard ferromagnetic phase with perpendicular magnetic anisotropy up to a transition temperature of $\approx 180 \mathrm{~K}$, where the ZFC $\chi_{\perp}$ attains a maximum. Indeed, at $5 \mathrm{~K}$ the hysteresis loop for perpendicular applied field shows a coercivity of $5.05 \mathrm{kOe}$, while for parallel field it shows none (Fig. 2). This perpendicular coercivity decreases with increasing temperature until it vanishes at $T_{1} \approx 200 \mathrm{~K}$ (Fig. 3), close to the maximum in $\chi_{\perp}$.

ii) An intermediate ordered magnetic phase without coercivity. The behaviour of $\chi$ is similar to that of an asperomagnetic phase as in spin-glass-like systems. The transition temperature $T_{1}$ between the ferromagnetic and asperomagnetic phases is defined by the cusp in $\chi$. At $T_{\mathrm{f}} \approx 375 \mathrm{~K}$ the magnetization of the NPs freezes along easy axis directions which are randomly distributed around an axis normal to the sample's plane.

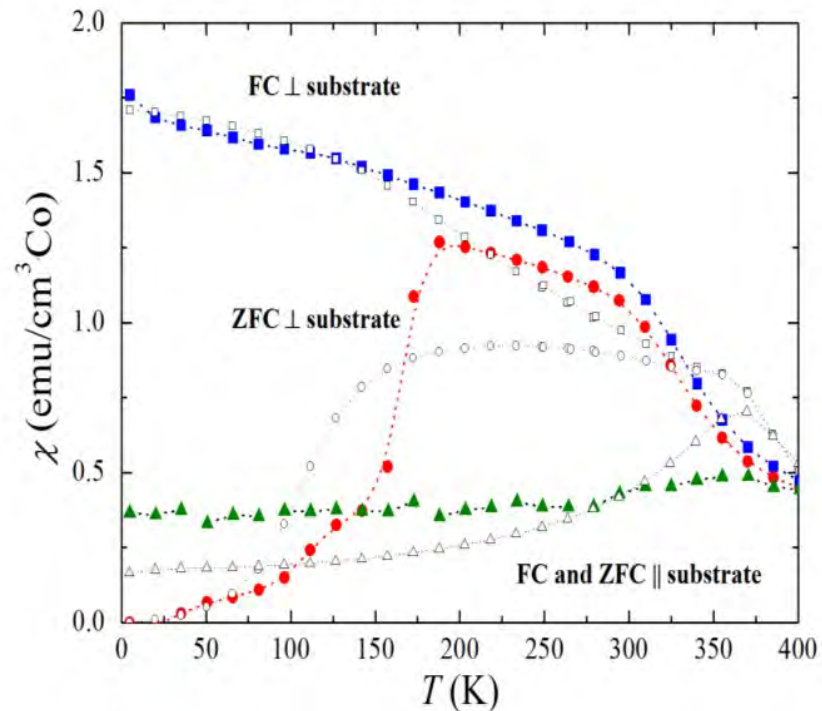

Figure 1. FC and ZFC magnetic susceptibility for applied field parallel and perpendicular to the sample's plane of the $\left[\mathrm{Al}_{2} \mathrm{O}_{3} / \mathrm{Co} / \mathrm{Pd}\right]$ single layer (full symbols) and $\left[\mathrm{Al}_{2} \mathrm{O}_{3} / \mathrm{Co} / \mathrm{Pd}\right]_{25}$ multilayer (open symbols) [8].

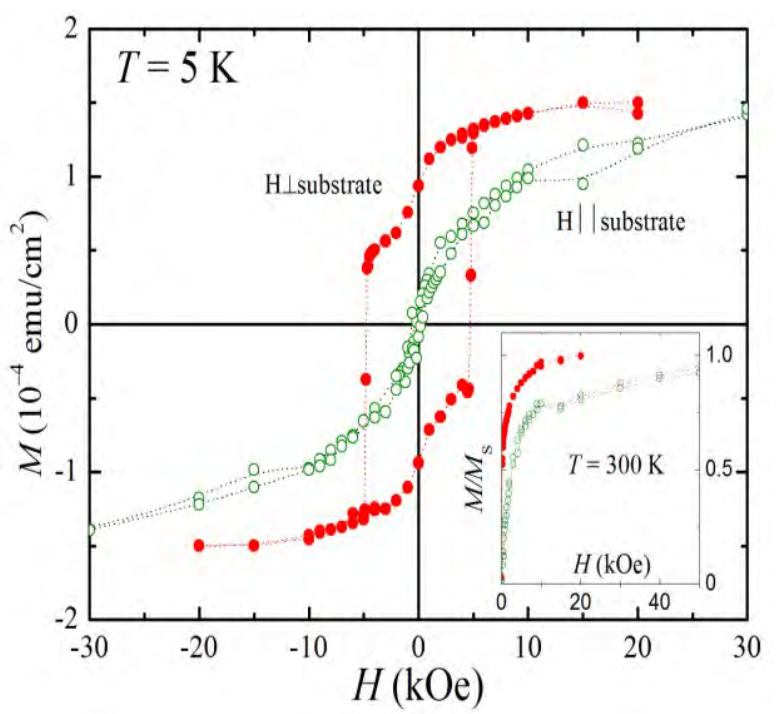

Figure 2. Hysteresis loops at $5 \mathrm{~K}$ for applied field parallel (open symbol) and perpendicular (full symbol) to the sample's plane. Inset: Reduced magnetization curve at $300 \mathrm{~K}$ for both directions of the applied field. 
iii) Beyond $T_{\mathrm{f}}$, perpendicular and parallel $\mathrm{FC}$ and $\mathrm{ZFC}$ curves merge, which marks the beginning of the superparamagnetic phase.

Since both the single layer and multilayer of CoPt NPs show the same sequence of magnetic phases at very similar transition temperatures, it can be concluded that interlayer magnetic coupling is negligible or absent in the multilayers. Moreover, it should be noticed that the asperomagnetic phase is already present in the single layer (i.e., the first layer in the multilayer system), where Co quasi-spherical NPs form a hexagonal array. Therefore, there is an intralayer distribution of easy axes produced at the NP level when Pt covers and alloys with the predeposited Co NPs. In fact, the reduced magnetization of this phase sharply mounts to $1 / 2$ at zero field (Fig. 2, inset), in agreement with the average value over a hemisphere of the projection of the magnetization onto the normal to the sample's plane, and then increases steadily for increasing magnetic field, as expected for a narrowing of the magnetization towards the cone axis.

The maximum in $\chi$, which defines the ferro-asperomagnetic phase transition, is neatly observed as a cusp in the single layer, while it is broad in the multilayer. This qualitative difference in $\chi$ can be ascribed to a decrease in the quality of the formation of the granular $\mathrm{Co} / \mathrm{Pt}$ layers as they are sequentially deposited, resulting in a broader distribution of easy axis directions than in the single layer sample.

The deposition of Pt on Co produces alloyed CoPt NPs with a core-shell structure [8]. While this was only slightly reflected in the $5 \mathrm{~K}$ hysteresis loops for the multilayers [8], it is clearly seen in the present single layer as a step in the $2^{\text {nd }}$ and $3^{\text {rd }}$ quadrants. This fact points towards a better structural formation of the CoPt NPs at the first $\left[\mathrm{Al}_{2} \mathrm{O}_{3} / \mathrm{Co} / \mathrm{Pt}\right]$ layer, in line with the behaviour of the ZFC susceptibility for perpendicular applied field mentioned above.

The $5 \mathrm{~K}$ coercivity of the single layer is $\approx$ $25 \%$ larger than in the sample with 25 layers. The asperomagnetic phase has no coercivity, but it shows perpendicular magnetic anisotropy (Fig. 2, inset) similarly to multilayers with $\mathrm{Pt}$ and $\mathrm{Pd}$ cappings [8-10], which also show the same sequence of magnetic phases, including the asperomagnetic-like one.

The hysteresis loops for magnetic field applied perpendicularly to the sample's plane were also measured using anomalous Hall effect and

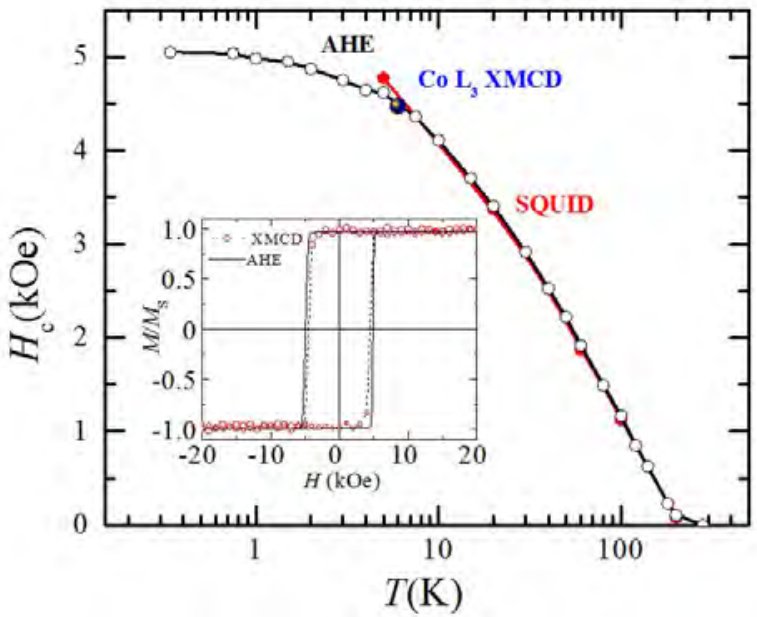

Figure 3. Temperature dependence of the coercivity for applied magnetic field perpendicular to the substrate's plane measured with a SQUID (full circles), AHE (open circles) and XMCD. Inset: Hysteresis loops obtained by XMCD at the Co $L_{3}$ edge at $6 \mathrm{~K}$, and $\mathrm{AHE}$ at $5 \mathrm{~K}$. XMCD spectra at the Co $L_{3}$ edge (Fig. 3, inset). Unlike the loops measured with the SQUID magnetometer, these do not show the core-shell structure of the NPs. Concerning the AHE loop, one may argue that the scattering by the magnetic Co atoms in the NP's shell could reduce the electron flux at the core, and consequently its contribution to AHE. However, we have no explanation for the behaviour of the XMCD loop since the FY detection method employed is bulk sensitive and should probe the NPs as a whole.

In summary, the magnetic study of a $\left[\mathrm{Al}_{2} \mathrm{O}_{3} / \mathrm{Co} / \mathrm{Pt}\right]$ single layer clearly reveals that there is no interlayer interaction in the $\left[\mathrm{Al}_{2} \mathrm{O}_{3} / \mathrm{Co} / \mathrm{Pt}\right]_{\mathrm{N}}$ multilayer. The CoPt alloy nanoparticles composing the layer show a core-shell structure, evidenced in SQUID hysteresis loops. The low temperature phase is ferromagnetic, brought about by intra-layer interactions of CoPt NPs, and shows perpendicular magnetic anisotropy. Above a transition temperature $T_{1}$, the phase is asperomagnetic, with NPs which still show PMA up to a freezing temperature $T_{\mathrm{f}}$. 


\section{Acknowledgements.}

The authors acknowledge the financial support of the Spanish Ministerio de Economía under the grant MAT2014-53921-R and of Aragonese grant DGA-IMANA E34. The use of Servicio General de Apoyo a la Investigación-SAI, Universidad de Zaragoza is also acknowledged. European Synchrotron Radiation Facility beamtime corresponds to HE2541 experiment.

\section{References}

[1] F. Luis, F. Petroff, J. M. Torres, L. M. García, J. Bartolomé, J. Carrey, and A. Vaurès, Phys. Rev. Lett. 88 (2002) 217205.

[2] J. Bartolomé, F. Luis, F. Petroff, F. Bartolomé, L.M.García, V. Cross and H. Jaffres, Phys. Met. Metallogr. 99, (2005) S8.

[3] J. Bartolomé, F .Bartolomé, L.M. García, F. Luis, F. Petroff, V. Cross, H. Jaffres, and A. Vaurès, in Smart Materials for Ranging Systems, edited by J. Franse, V. Eremenko, and V. Sirenko, Nato Science Series II Vol. 226 (Springer, Netherlands, 2006), pp. 1-25.

[4] F. Luis, F. Bartolome, F. Petroff, J. Bartolomé, L.M. García, C. Deranlot, H. Jaffres, M. J. Martínez, P. Bencok, F. Wilhelm, A. Rogalev and N. Brookes, Europhys. Lett. 76 (2006) 142 (2006).

[5] J. Bartolomé, L.M.García, F. Bartolomé , F. Luis, R. López-Ruiz, F. Petroff, C. Deranlot, F. Wilhelm, A. Rogalev, P. Bencok, N. B. Brookes, L. Ruiz, and J. M. González-Calbet, Phys. Rev. B 77 (2008) 184420.

[6] A. I. Figueroa, J. Bartolomé, L. M. García, F. Bartolomé, C. Magén, A. Ibarra, L. Ruiz, J. M. González-Calbet, F. Petroff, C. Deranlot, S. Pascarelli, P. Bencok, N. B. Brookes, F. Wilhelm, and A. Rogalev, Phys. Rev. B 84 (2011) 184423.

[7] J. Bartolomé, A. I. Figueroa, L. M. García, F. Bartolomé, L. Ruiz , J. M. González-Calbet , F. Petroff, C Deranlot, F. Wilhelm, A. Rogalev and N. Brookes, Low Temp. Phys. 38 (2012) 835 (2012).

[8] A.I. Figueroa, J. Bartolomé, L.M. García, F. Bartolomé, O. Bunau, J. Stankiewicz, L Ruiz, J.M. González-Calbet, F. Petroff, C. Deranlot, S. Pascarelli, P. Bencok, N.B. Brookes, F. Wilhelm, A. Smekhova and A. Rogalev, Phys. Rev. B 90 (2014) 174421(16).

[9] L.G. Vivas, A.I. Figueroa, F. Bartolomé, J. Rubín, L.M. García, C. Deranlot, F. Petroff, L Ruiz, J.M. González-Calbet, N.B. Brookes, F. Wilhelm, A. Rogalev, J. Bartolomé, J. Magn. Magn. Mat. 400 (2016) 248-252.

[10] L.G. Vivas, J. Rubín, A.I. Figueroa, F. Bartolomé, L.M. García, C. Deranlot, F. Petroff, L Ruiz, J.M. González-Calbet, S. Pascarelli, N.B. Brookes, F. Wilhelm, A. Rogalev, J. Bartolomé, Phys. Rev. B 93 (2016) 174410(18). 